\title{
Genome-wide association study identifies an early onset pancreatic cancer risk locus
}

Daniele Campa $^{1}$ ( ) | Manuel Gentiluomo ${ }^{1}$ ( ) | Ofure Obazee $^{2}$ | Alba Ballerini $^{1}$ | Ludmila Vodickova $^{3,4}$ | Péter Hegyi ${ }^{5,6}$ | Pavel Soucek ${ }^{7}$ (i) | Hermann Brenner ${ }^{8,9,10}$ | Anna Caterina Milanetto ${ }^{11}$ | Stefano Landi ${ }^{1}$ | Xin Gao ${ }^{8}$ | Dania Bozzato ${ }^{12}$ | Gabriele Capurso $^{13,14}$ | Francesca Tavano ${ }^{15}$ [ | Yogesh Vashist ${ }^{16}$ | Thilo Hackert $^{17}$ | Franco Bambi ${ }^{18}$ | Simona Bursi ${ }^{19}$ | Martin Oliverius ${ }^{20}$ | Domenica Gioffreda $^{15}$ | Ben Schöttker ${ }^{8}$ () | Audrius Ivanauskas ${ }^{21}$ | Beatrice Mohelnikova-Duchonova $^{22}$ | Erika Darvasi $^{6}$ | Raffaele Pezzilli ${ }^{23}$ | Ewa Małecka-Panas $^{24}$ | Oliver Strobel ${ }^{17}$ | Maria Gazouli ${ }^{25}$ ( ) | Verena Katzke ${ }^{26}$ | Andrea Szentesi ${ }^{5,6}$ | Giulia Martina Cavestro ${ }^{27}$ | Gyula Farkas $\mathrm{Jr}^{28}$ | Jakob R. Izbicki ${ }^{16}$ | Stefania Moz ${ }^{12}$ | Livia Archibugi ${ }^{13,14}$ | Viktor Hlavac ${ }^{29}$ | Áron Vincze $^{30}$ | Renata Talar-Wojnarowska ${ }^{24}$ | Borislav Rusev ${ }^{31}$ | Juozas Kupcinskas $^{21}$ | Bill Greenhalf ${ }^{32}$ | Frederike Dijk ${ }^{33}$ | Nathalia Giese ${ }^{17}$ | Ugo Boggi $^{34}$ | Angelo Andriulli ${ }^{15}$ | Olivier R Busch ${ }^{35}$ | Giuseppe Vanella ${ }^{13}$ | Pavel Vodicka $^{3,4}$ | Michael Nentwich ${ }^{16}$ | Rita T. Lawlor ${ }^{31}$ | George E Theodoropoulos $^{36}$ | Krzysztof Jamroziak ${ }^{37}$ | Raffaella Alessia Zuppardo ${ }^{27}$ | Lucia Moletta $^{11}$ | Laura Ginocchi ${ }^{19}$ | Rudolf Kaaks ${ }^{26}$ ( John P Neoptolemos ${ }^{17}$ | Maurizio Lucchesi ${ }^{19}$ | Federico Canzian ${ }^{2}$ ()

\footnotetext{
${ }^{1}$ Department of Biology, University of Pisa, Pisa, Italy

${ }^{2}$ Genomic Epidemiology Group, German Cancer Research Center (DKFZ), Heidelberg, Germany

${ }^{3}$ First Faculty of Medicine, Institute of Biology and Medical Genetics, Charles University, Prague, Czech Republic

${ }^{4}$ Biomedical Centre, Faculty of Medicine in Pilsen, Charles University in Prague, Pilsen, Czech Republic

${ }^{5}$ Institute for Translational Medicine, Medical School, University of Pécs, Pécs, Hungary

${ }^{6}$ First Department of Medicine, University of Szeged, Szeged, Hungary

${ }^{7}$ Biomedical Center, Faculty of Medicine in Pilsen, Charles University, Pilsen, Czech Republic

${ }^{8}$ Division of Clinical Epidemiology and Aging Research, German Cancer, Research Center (DKFZ), Heidelberg, Germany

${ }^{9}$ Division of Preventive Oncology, German Cancer Research Center (DKFZ) and National Center for Tumor Diseases (NCT), Heidelberg, Germany

${ }^{10}$ German Cancer Consortium (DKTK), German Cancer Research Center (DKFZ), Heidelberg, Germany

${ }^{11}$ Department of DISCOG, University of Padova, Padova, Italy

${ }^{12}$ Department of DIMED, University of Padova, Padova, Italy

${ }^{13}$ Digestive and Liver Disease Unit, S. Andrea Hospital, University Sapienza, Rome, Italy

${ }^{14}$ Pancreatico/Biliary Endoscopy and Endosonography Division, Pancreas Translational and Clinical Research Center, San Raffaele Scientific Institute, Milan, Italy
}

Abbreviations: EOPC, early onset pancreatic cancer; eQTLs, expression quantitative trait loci; GWAS, genome-wide association study; LD, linkage disequilibrium; MAF, minor allele frequency; NEOPC, Non-EOPC; OR, odds ratio; P, P-value or probability value; PanC4, Pancreatic Cancer Case-Control Consortium; PANDoRA, PANcreatic Disease ReseArch; PDAC, pancreatic ductal adenocarcinoma; VEOPC, very early onset pancreatic cancer. 
${ }^{15}$ Division of Gastroenterology and Research Laboratory, Fondazione "Casa Sollievo della Sofferenza” Hospital, I.R.C.C.S, San Giovanni Rotondo, Italy

${ }^{16}$ Department of General Visceral and Thoracic Surgery, University Clinic Hamburg-Eppendorf, Hamburg, Germany

${ }^{17}$ Department of General, Visceral and Transplantation Surgery, University of Heidelberg, Heidelberg, Germany

${ }^{18}$ Blood Transfusion Service, Azienda Ospedaliero-Universitaria Meyer, Florence, Italy

${ }^{19}$ Oncological Department, Azienda USL Toscana Nord Ovest, Oncological Unit of Massa Carrara, Carrara, Italy

${ }^{20}$ Department of Surgery, Faculty Hospital Kralovske Vinohrady and Third Faculty of Medicine, Charles University, Prague, Czech Republic

${ }^{21}$ Department of Gastroenterology and Institute for Digestive Research, Lithuanian University of Health Sciences, Kaunas, Lithuania

${ }^{22}$ Department of Oncology and Institute of Molecular and Translational Medicine, Faculty of Medicine and Dentistry, Palacky University and University Hospital, Olomouc, Czech Republic

${ }^{23}$ Pancreas Unit, Department of Gastroenterology, Polyclinic of Sant'Orsola, Bologna, Italy

${ }^{24}$ Department of Digestive Tract Diseases, Medical University of Lodz, Lodz, Poland

${ }^{25}$ Laboratory of Biology, Medical School, National and Kapodistrian University of Athens, Athens, Greece

${ }^{26}$ Division of Cancer Epidemiology, German Cancer Research Center (DKFZ), Heidelberg, Germany

${ }^{27}$ Gastroenterology and Gastrointestinal Endoscopy Unit, Vita-Salute San Raffaele University, San Raffaele Scientific Institute, Milan, Italy

${ }^{28}$ Department of Surgery, University of Szeged, Szeged, Hungary

${ }^{29}$ Department of Toxicogenomics, National Institute of Public Health, Prague, Czech Republic

${ }^{30}$ Division of Translational Medicine, First Department of Medicine, Medical School, University of Pécs, Pécs, Hungary

${ }^{31}$ ARC-Net Research Centre, University and Hospital Trust of Verona, Verona, Italy

${ }^{32}$ Molecular and Clinical Cancer Medicine, Royal Liverpool University Hospital, Liverpool, UK

${ }^{33}$ Department of Pathology, Amsterdam UMC, University of Amsterdam, Amsterdam, The Netherlands

${ }^{34}$ Division of General and Transplant Surgery, Pisa University Hospital, Pisa, Italy

${ }^{35}$ Department of Surgery, Cancer Center Amsterdam, Amsterdam UMC, University of Amsterdam, Amsterdam, The Netherlands

${ }^{36} 1$ st Propaedeutic University Surgery Clinic, Hippocratio General Hospital, Medical School, National and Kapodistrian University of Athens, Athens, Greece

${ }^{37}$ Department of Hematology, Institute of Hematology and Transfusion Medicine, Warsaw, Poland

\section{Correspondence}

Daniele Campa, Department of Biology, University of Pisa, Via Derna 1, 56126 Pisa, Italy.

Email: daniele.campa@unipi.it

\section{Funding information}

Charles University project "Center of clinical and experimental liver surgery", Grant/Award Number: UNCE/MED/006; "5×1000" voluntary contribution; Associazione Italiana per la Ricerca sul Cancro (AIRC), Grant/Award Number: IG 17177; Baden-Württemberg state Ministry of Science, Research and Arts; Economic Development and Innovation Operative Programme Grant, Grant/Award Number: GINOP 2.3.2-15-2016-00048; Fondazione Arpa; Fondazione Tizzi; Hungarian Academy of Sciences, Grant/Award Number: LP2014-10/2014; Italian Ministry of Health, Grant/Award Number: RC1803GA32; Ministry of Health of the Czech Republic, Grant/Award Number: NV19-03-00097; Ministry of Health of the Czech Republic DRO, Grant/Award Number: 00098892

\section{Abstract}

Early onset pancreatic cancer (EOPC) is a rare disease with a very high mortality rate. Almost nothing is known on the genetic susceptibility of EOPC, therefore, we performed a genome-wide association study (GWAS) to identify novel genetic variants specific for patients diagnosed with pancreatic ductal adenocarcinoma (PDAC) at younger ages. In the first phase, conducted on 821 cases with age of onset $\leq 60$ years, of whom 198 with age of onset $\leq 50$, and 3227 controls from PanScan I-II, we observed four SNPs (rs7155613, rs2328991, rs4891017 and rs12610094) showing an association with EOPC risk $\left(P<1 \times 10^{-4}\right)$. We replicated these SNPs in the PANcreatic Disease ReseArch (PANDoRA) consortium and used additional in silico data from PanScan III and PanC4. Among these four variants rs2328991 was significant in an independent set of 855 cases with age of onset $\leq 60$ years, of whom 265 with age of onset $\leq 50$, and 4142 controls from the PANDoRA consortium while in the in silico data, we observed no statistically significant association. However, the resulting meta-analysis supported the association $\left(P=1.15 \times 10^{-4}\right)$. In conclusion, we propose a novel variant rs2328991 to be involved in EOPC risk. Even though it was not possible to find a mechanistic link between the variant and the function, the association is supported by a solid statistical significance obtained in the largest study on EOPC genetics present so far in the literature.

\section{KEYWORDS}

early onset, genome-wide association study, pancreatic cancer, single nucleotide polymorphisms, very early onset pancreatic cancer 


\section{1 | INTRODUCTION}

Pancreatic cancer is the fifth most common cause of cancer death worldwide ${ }^{1}$ and it is projected to become the second by $2030 .^{2}$ Several epidemiologic factors show a strong effect on pancreatic cancer susceptibility including smoking, alcohol consumption and obesity. ${ }^{3}$ In the last 10 years, almost 30 sporadic pancreatic cancer risk loci have been identified through genome wide association studies (GWAS) and large scale candidate gene studies. ${ }^{4-15}$

The median age of onset of pancreatic cancer is 71 years and around $20 \%$ of subjects develop it before 60 years of age, defined as early onset pancreatic cancer (EOPC). ${ }^{16-19}$ Only around $3 \%$ of cases are diagnosed before 45 years of age (very early onset pancreatic cancer, VEOPC). ${ }^{17}$ EOPC and VEOPC share the majority of epidemiologic risk factors with non-EOPC (NEOPC), with smoking being the strongest for both ages of onset. ${ }^{16,17}$ Not much is known on the genetic background of EOPC, however in a recent report BenAharon and colleagues comparing the somatic mutation landscape of NEOPC and EOPC, found several differences in the pathways involved. ${ }^{20}$ In addition, only one study has been performed to identify germline single nucleotide polymorphisms (SNPs) specifically associated with EOPC. ${ }^{21}$ In that manuscript, Chen et al identified eight SNPs associated with an earlier diagnosis. A better understanding of the genetic background could be extremely helpful in identifying molecular pathways that could lead to early carcinogenesis and therefore expand our understanding on the disease. With these premises, we performed a GWAS on EOPC with the aim of identifying novel variants specific for younger pancreatic ductal adenocarcinoma (PDAC) patients.

\section{2 | MATERIALS AND METHODS}

\subsection{Populations used in the study}

We used a two-phase approach: in the discovery phase, we have used the Pancreatic Cancer Cohort Consortium (PanScan) study that has been fully described elsewhere. ${ }^{4,8}$ Briefly, case and control data and DNA samples were collected from 12 cohort studies and 8 casecontrol studies. Cases were defined as those individuals having primary adenocarcinoma of the exocrine pancreas. Controls were frequency matched to cases and were free of pancreatic cancer at the time of enrolment. Matching criteria varied according to the studies within PanScan I-II. We analyzed 3133 PDAC patients among whom there were 821 cases with the age of onset $\leq 60$ years and 198 with the age of onset $\leq 50$, and 3227 controls. For replication, we used three populations: phase three of the PanScan (PanScan III) study, ${ }^{10}$ the Pancreatic Cancer Case-Control Consortium (PanC4) ${ }^{12}$ and the PANcreatic Disease ReseArch (PANDoRA) consortium. ${ }^{22}$ For PanScan III and PanC4, the replication was done "in silico" using data from PANDoRA includes studies from nine European countries in which cases were defined by an established diagnosis of PDAC and controls were individuals from the general population without a

\section{What's new?}

Early-onset pancreatic cancer (EOPC), diagnosed between ages 45 and 60, accounts for about one-fifth of all pancreatic cancers. Nonetheless, while multiple epidemiological risk factors for EOPC have been identified, very little is known about genetic susceptibility. The present genome-wide association study identifies four novel single nucleotide polymorphisms specific for patients diagnosed with pancreatic cancer at younger ages. Among the variants, 13q22.3_rs2328991 was associated with elevated risk in an independent set of pancreatic cancer patients, some of whom experienced disease onset at age 50 or younger. The findings highlight a need for further research on the genetics of EOPC.

pancreatic disease at recruitment, individuals who were hospitalized for nontumor related causes, or blood donors. Table 1 summarizes the subjects used for our study. We validated the results with a total of 8096 cases (695 younger than 50 and 2385 younger than 60) and 7741 controls.

\section{2 | Data filtering, sample preparation and genotyping}

For the first phase (PanScan I-II), we downloaded the genotyping data from the database of Genotypes and Phenotypes ( $\mathrm{dbGaP}$, study accession number phs000206.v5.p3, project reference \#12644). We used 60 and 50 years of age as thresholds to define groups of EOPC cases. Ages are coded in 10-year intervals in data downloaded from dbGaP (eg, 40-50, 50-60, etc.), therefore, we were unable to analyze only cases younger than 45 , which correspond to the exact definition of VEOPC. The validation in PanScan III and PanC4 was done in silico using data from dbGaP. For all datasets obtained from dbGaP, genotyping procedures, genotyping quality control checks, data collection were thoroughly reported in the original publications. ${ }^{4,6,8,10}$ We removed individuals with gender mismatches, call rate $<0.9$, minimal or excessive heterozygosity ( $>3$ SD from the mean) or cryptic relatedness (PI_HAT>0.2). We discarded SNPs with a minor allele frequency $<0.5 \%$, completion rate $<90 \%$, evidence for violations of Hardy-Weinberg Equilibrium $\left(P<10^{-6}\right)$. Principal component analysis was carried out including genotypes from all the populations of the phase 3 of the 1000 Genomes Project (http://www.internationalgenome.org/). Individuals not clustering with the 1000 Genomes subjects of European descent were excluded from further analysis.

We performed de novo genotyping for PANDoRA. DNAs were extracted from blood samples using the Qiagen mini kit (Qiagen, Hilden, Germany) at the German Cancer Research Center (DKFZ) in Heidelberg, according to the manufacturer's protocol. DNA concentration was measured using spectrophotometer and samples were 
TABLE 1 Description of the study population

\begin{tabular}{|c|c|c|c|c|}
\hline Study phase & Country/study & Cases & Controls & Total \\
\hline \multirow{10}{*}{$\begin{array}{l}\text { Phase } 1 \\
\text { (PanScan) }\end{array}$} & PanScan I & 2040 & 2048 & 4088 \\
\hline & PanScan II & 1093 & 1179 & 2272 \\
\hline & Total & 3133 & 3227 & 6360 \\
\hline & Sex & & & \\
\hline & Male & $52.9 \%$ & $53.2 \%$ & $53.0 \%$ \\
\hline & Female & $47.1 \%$ & $46.8 \%$ & $47.0 \%$ \\
\hline & Age & & & \\
\hline & Median, years & 65 & 65 & 65 \\
\hline & $\leq 50, \mathrm{~N}$ & 198 & 217 & 415 \\
\hline & $\leq 60, \mathrm{~N}$ & 821 & 800 & 1621 \\
\hline \multirow{17}{*}{$\begin{array}{l}\text { Phase } 2 \\
\text { (PANDoRA) }\end{array}$} & Czech Republic & 347 & 431 & 778 \\
\hline & Germany & 839 & 1708 & 2547 \\
\hline & Greece & 114 & 16 & 130 \\
\hline & Hungary & 259 & 332 & 591 \\
\hline & Italy & 792 & 1282 & 2074 \\
\hline & Lithuania & 57 & 189 & 246 \\
\hline & Netherlands & 117 & 0 & 117 \\
\hline & Poland & 99 & 184 & 283 \\
\hline & United Kingdom & 87 & 0 & 87 \\
\hline & Total & 2711 & 4142 & 6853 \\
\hline & Sex & & & \\
\hline & Male & $56 \%$ & $54 \%$ & $55 \%$ \\
\hline & Female & $44 \%$ & $46 \%$ & $45 \%$ \\
\hline & Age & & & \\
\hline & Median, years & 64 & 56 & 59 \\
\hline & $\leq 50, \mathrm{~N}$ & 265 & 1256 & 1521 \\
\hline & $\leq 60, \mathrm{~N}$ & 855 & 2505 & 3360 \\
\hline \multirow{16}{*}{$\begin{array}{l}\text { Phase } 2 \\
\text { (PanScan } \\
\text { PanC4) }\end{array}$} & PanScan III & 1522 & - & 1522 \\
\hline & Sex & & & \\
\hline & Male & $49 \%$ & - & $49 \%$ \\
\hline & Female & $51 \%$ & - & $51 \%$ \\
\hline & Age & & & \\
\hline & Median, years & 69 & - & 69 \\
\hline & $\leq 50, N$ & 76 & - & 76 \\
\hline & $\leq 60, \mathrm{~N}$ & 354 & - & 354 \\
\hline & PanC4 & 3863 & 3599 & 7462 \\
\hline & Sex & & & \\
\hline & Male & $58 \%$ & $56 \%$ & $57 \%$ \\
\hline & Female & $42 \%$ & $44 \%$ & $43 \%$ \\
\hline & Age & & & \\
\hline & Median, years & 65 & 64 & 65 \\
\hline & $\leq 50, \mathrm{~N}$ & 354 & 375 & 729 \\
\hline & $\leq 60, N$ & 1176 & 1277 & 1176 \\
\hline
\end{tabular}

kept frozen till genotyping. Genotyping was performed using TaqMan technology assays as recommended by the manufacturer in 384 well plates using $10 \%$ of samples as duplicates for quality control purposes.
We observed no deviation from Hardy-Weinberg equilibrium in the controls, an average call rate $>99 \%$ and a concordance with the duplicated samples of $99.2 \%$.

\section{3 | Statistical analysis}

In phase one, we performed a GWAS on EOPC risk, analyzing 630600 SNPs with unconditional logistic regression, adjusted for study, sex, two main principal components and study. We performed four analyses: (a) considering individuals $\leq 50$ years when diagnosed with PDAC vs all controls, (b) individuals $\leq 60$ years when diagnosed with PDAC vs all controls, (c) a case-case analysis considering cases $\leq 50$ vs older cases and (d) cases $\leq 60$ vs older cases. For all analyses, we used an allelic, a dominant and a recessive inheritance model, using the more common allele in the controls as reference. All non-Caucasian individuals were excluded from the analyses.

We replicated in the second phase all SNPs that reached a $P$-value of at least $1 \times 10^{-4}$ in at least one of the analyses done. Data from PanScan III was used for replication of the case-case analysis (PanScan III consists of only cases), while data from PanC4 and PANDoRA was used for all the analyses. Hardy-Weinberg equilibrium was checked for the SNPs that were genotyped in phase two of the study using Pearson exact chi square. In the second phase, analyses were also performed with unconditional logistic regression adjusting for sex and the two principal components (PanScan III and PanC4) or sex and country of origin (PANDoRA).

We performed two meta-analyses calculating heterogeneity between the studies (one for the case-control and one for the casecase analyses) using both fixed effect model and random effect model (depending on the heterogeneity) between phase one and phase two with a final sample size of 3206 EOPC, 893 cases with an onset before 50 years, 11229 total PDAC cases and 10908 controls.

In addition, to compare our data with the paper by Chen et al, we also checked the PanScan data for the SNP significant in their analysis. For none of the 8 SNPs reported by Chen et al, there were genotyping data in PanScan. However, for three of them (chr20_rs2766669, chr11_rs12803915, chr6_rs1559849), we found a SNP in LD $\left(r^{2}>0.80\right)$ that we used as surrogate in the analysis.

\section{4 | Bioinformatic analysis}

To understand the functional relevance of the SNPs significant after the second phase, we used RegulomeDB (http://regulome.stanford. edu/) ${ }^{23}$ and HaploReg v4.1 (https://pubs.broadinstitute.org/ mammals/haploreg/haploreg.php) ${ }^{24}$ to identify the regulatory potential of the region nearby the SNPs. The Genotype-Tissue Expression (GTEx) project (https://www.gtexportal.org/25 was used to identify potential eQTLs. Finally, we used SNP Nexus (https://snp-nexus. org $)^{26}$ as a database for functional annotation of SNPs. 


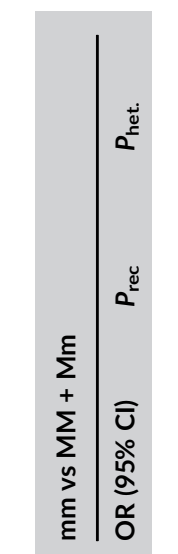

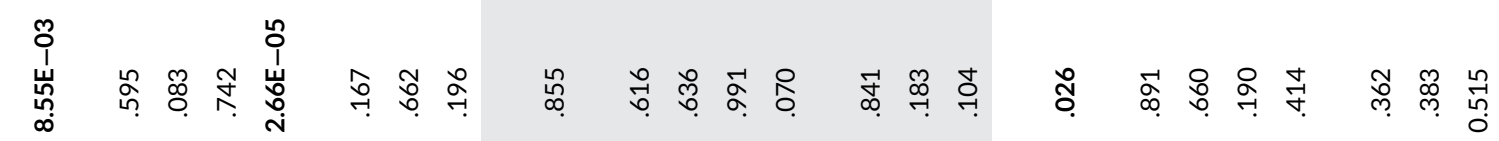

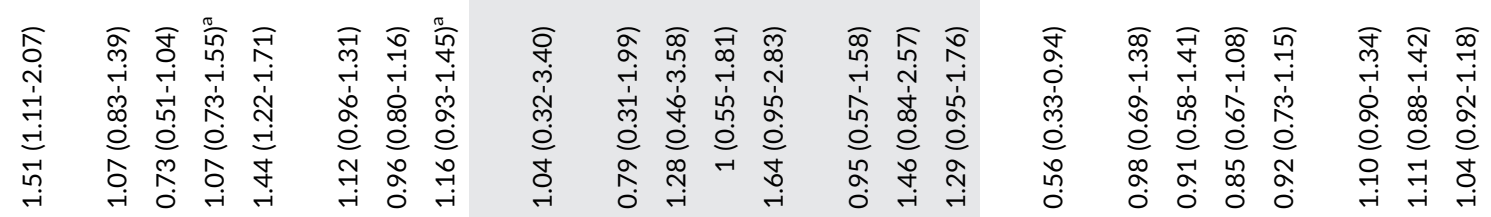

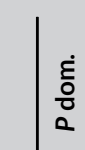

ڤ.

\%

F

ํ.

ธุง

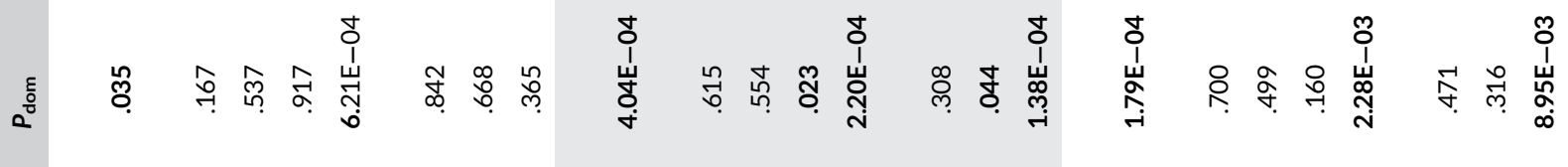

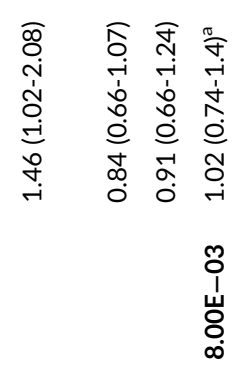

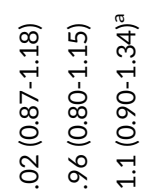

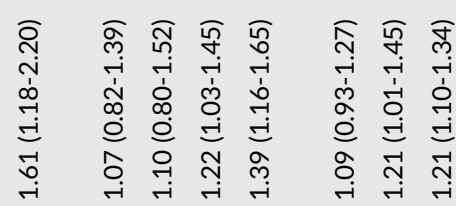

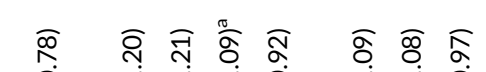

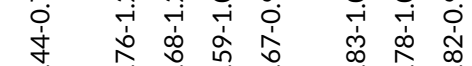

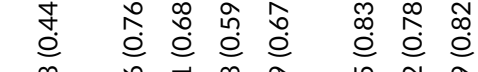

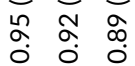

$\mid$

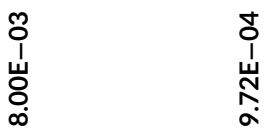

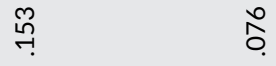

范

$\stackrel{\infty}{\text { స̆ }}$

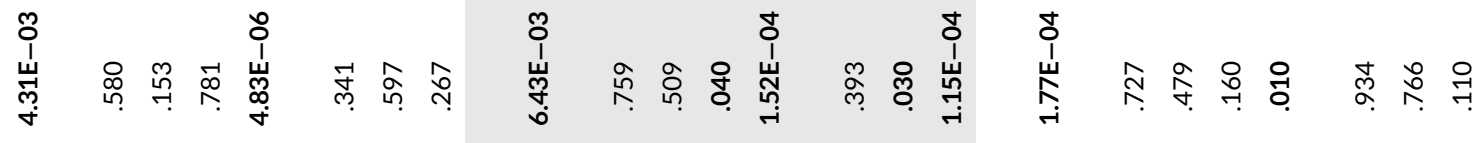

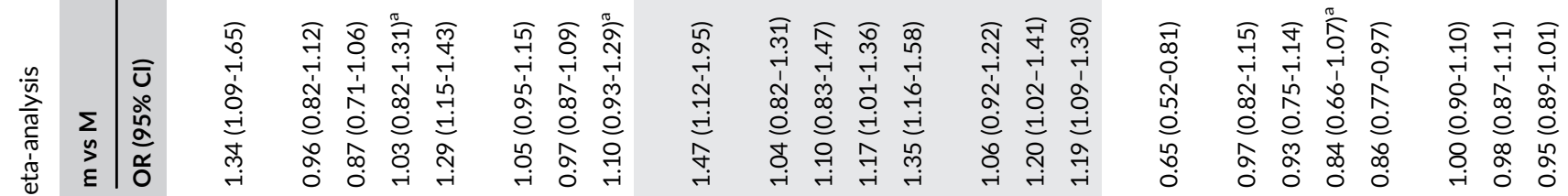

焉

든

这

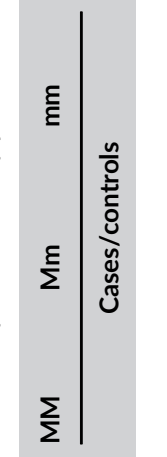

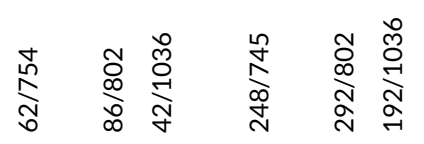

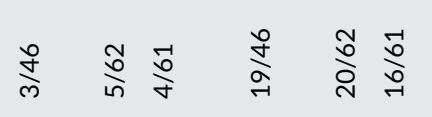

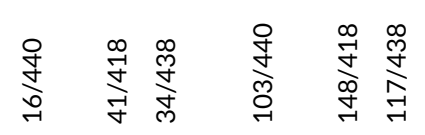

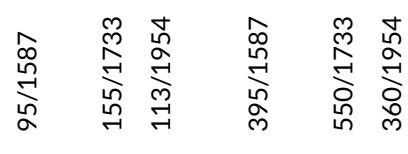

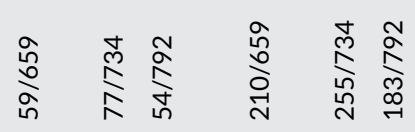

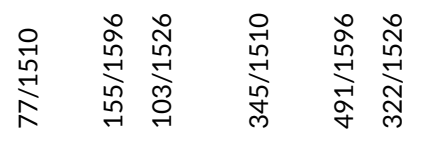

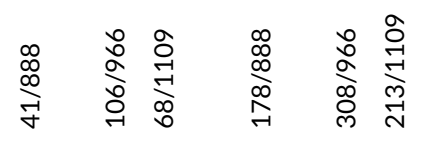

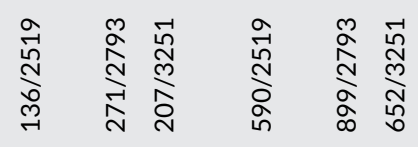

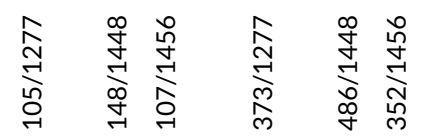

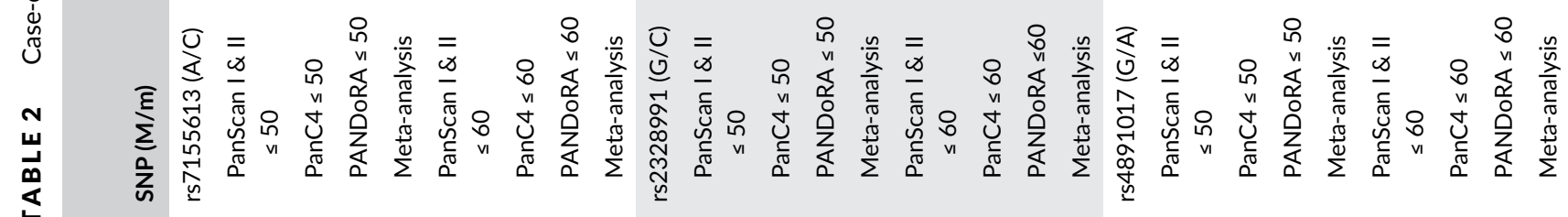



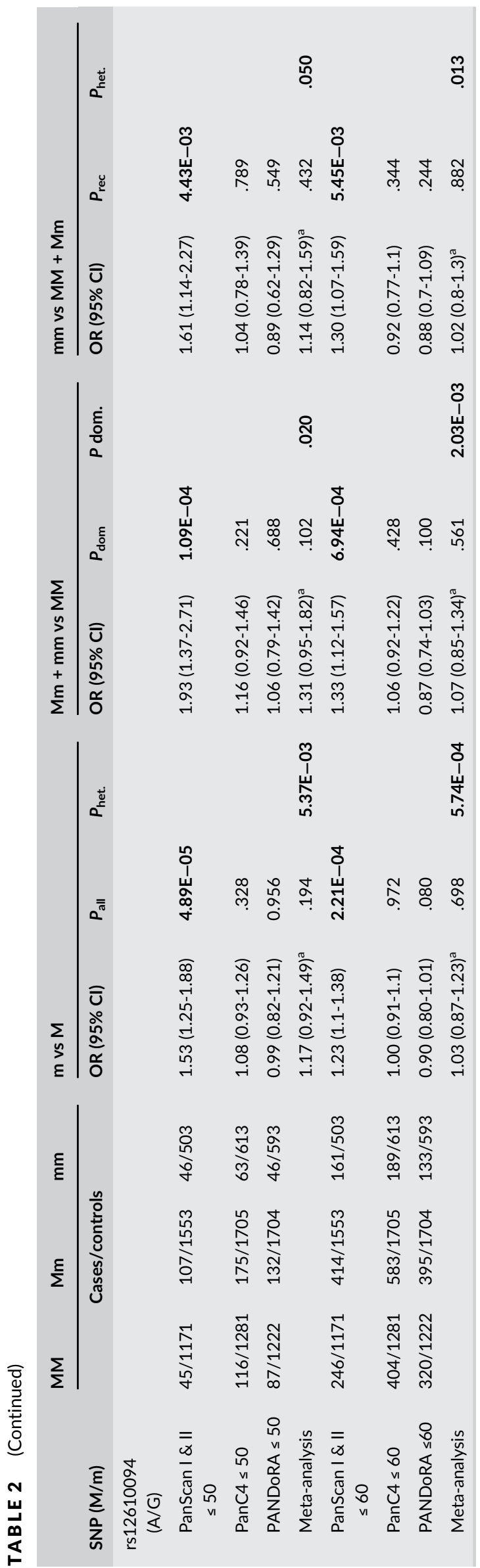

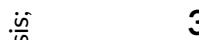

\section{3 | RESULTS}

In the first phase, which was conducted at a genome-wide scale on 3133 PDAC patients (among which 821 cases with the age of onset $\leq 60$ years and 198 with the age of onset $\leq 50$ ) and 3227 controls we observed four SNPs (14q24.3_rs7155613, 13q22.3_rs2328991, ZNF516_rs4891017 and OR7G2_rs12610094) that showed an association with EOPC risk with $P<1 \times 10^{-4}$ in the case-control analyses and/or in the case-case analyses.

We genotyped these SNPs in the PANDoRA consortium and used additional in silico data from PanScan III and PanC4. In the in silico data, we observed no statistically significant association in the selected SNPs. In PANDoRA, instead, using cases younger than 60 and all controls, we observed that carriers of the rare allele (C) of the 13q22.3_rs2328991 SNP showed a nominally statistically significant association with increased risk of developing EOPC (OR $=1.20$, $95 \% \mathrm{Cl} 1.02-1.41, P=3.00 \times 10^{-2}$ ) even though if considering multiple testing correction $(P<.05 / 4=0.0125)$ this association was not significant. In the meta-analysis between phase one and phase two of the study (PanScan I-II, PanC4, PANDoRA), we observed an association between the 13q22.3_rs2328991 $\mathrm{C}$ allele and an increased risk of developing EOPC (OR $=1.19,95 \% \mathrm{Cl}$ 1.09-1.30, $P=1.15$ $\left.\times 10^{-4}\right)$ with no heterogeneity $(P>.05)$. The results of the casecontrol analyses are shown in Table 2 . In the case-case analysis of the second phase alone, using 60 years as age cutoff, in PANDoRA, we observed that the minor allele (C) of the 13q22.3_rs2328991 showed a tendency of being associated with younger age of onset of PDAC reaching a statistically significant association (considering a threshold of $P<.05)$ in the recessive model of inheritance $\left(O R=2.06,95 \% \mathrm{Cl} 1.05-4.05, P=3.50 \times 10^{-2}\right)$. The case-case analyses also showed an association between 14q24.3_rs7155613 and age of onset of the disease $(\mathrm{OR}=0.81,95 \% \mathrm{Cl} 0.67-0.98, P=3.30 \times$ $10^{-2}$ ). PanScan III and PanC4 analysis did not show any statistically significant associations. The case-case meta-analysis showed significant results for all the four SNPs with the exception of 14q24.3_rs7155613. The results of the case-case analyses are shown in Table 3.

In addition, we also checked in PanScan the possible associations with EOPC risk of the eight variants identified by Chen and colleagues. ${ }^{21}$ We could find only three of the eight variants in the PanScan database and we observed no statistically significant association with EOPC risk.

\section{1 | Bioinformatic analysis}

Using HaploReg we found that rs2328991 is in strong linkage disequilibrium (LD) with 6 SNPs (rs17355129, rs7322104, rs76406862, rs80009395, rs79737810, rs76655255) in the Caucasian population and that is in a DNAse sensitive region. The bioinformatic tool does not show report any eQTLs for any of the SNPs. Regulome DB shows a score of 4 for rs2328991, a score of 5 for rs17355129 and rs76655255 a score of 6 for rs79737810 and no data for all other 


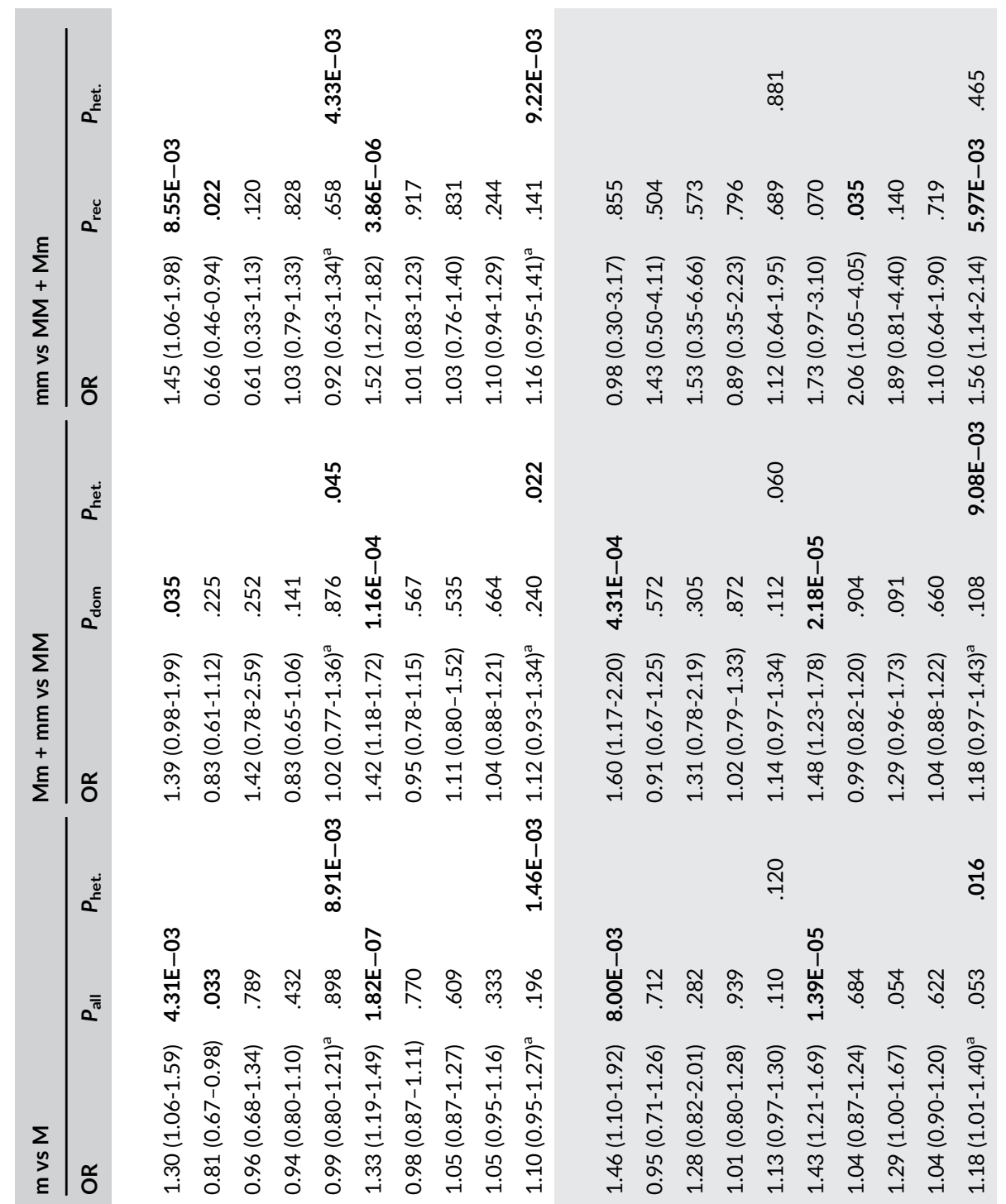

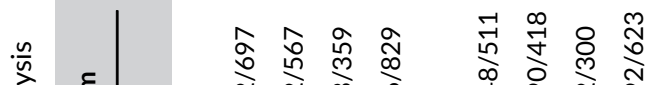

政

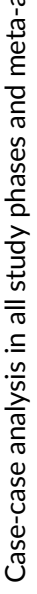

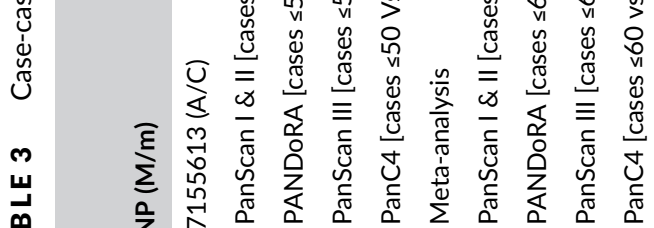

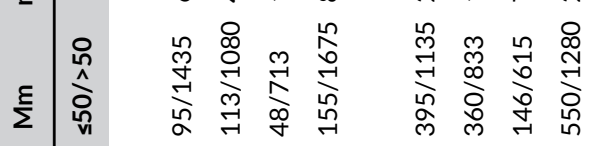

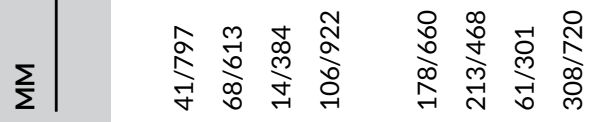

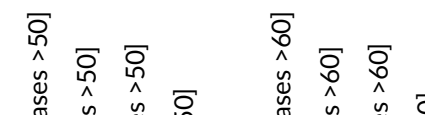

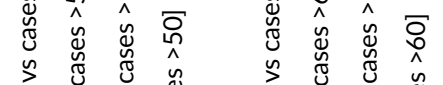

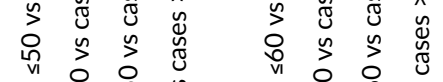

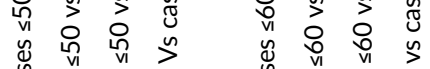

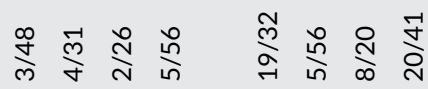

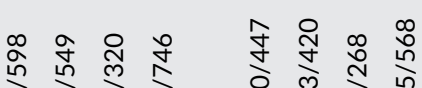
in

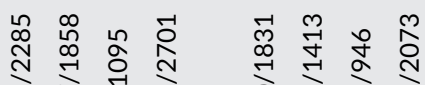

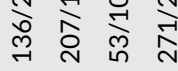
容㲾 


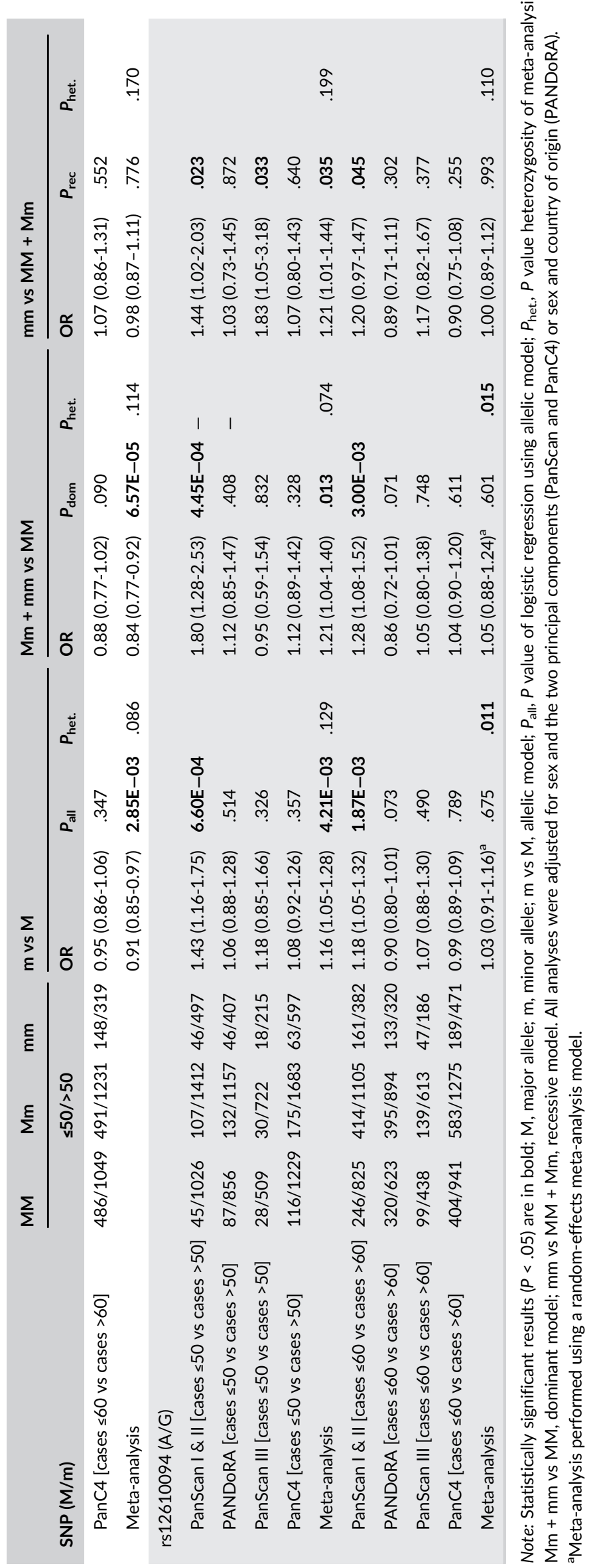


SNPs. Scores ranging from 6 to 4 represent a minimal binding evidence. The GTEx project does not show any statistically significant eQTLs for any of the selected SNPs.

\section{4 | DISCUSSION}

EOPC is a rare disease with a very high mortality rate, for which very few specific risk factors have been identified. ${ }^{17}$ Only a small number of genetic variants have been suggested in this regard. ${ }^{21}$ In this report, we aimed at uncovering novel polymorphisms associated with the disease. Our results suggest a potential involvement of 13q22.3_rs2328991 that is associated independently in PanScan I-II, PANDoRA and also in the meta-analysis with the same direction of the association. The minor allele of the SNP is associated with an increased chance of developing EOPC in the case-control analysis and also it is also associated with an increased chance of developing EOPC in comparison with NEOPC. The SNP however is associated with the disease in only one of the populations used for replication.

None of our results reached genome-wide statistical significance $\left(P<5 \times 10^{-8}\right)$ in either phase or in the meta-analysis. The lowest $P$-value we observed for the meta-analysis of PanScan, PanC4 and PANDoRA data is $1.15 \times 10^{-4}$ for the association of 13q22.3_rs2328991 with risk of pancreatic cancer under 60 years. This observation therefore has to be considered suggestive. However, the concordance of the results between two independent phases of our study is encouraging. No association, including that of 13q22.3_rs2328991, was consistently observed when considering cases diagnosed under 50 years of age. This is likely a reflection of the small numbers of cases in this category.

13q22.3_rs2328991 is situated $57 \mathrm{~kb}$ at the $3^{\prime}$ end of the potassium channel tetramerization domain containing 12 (KCTD12, OMIM no. 610521$)$. In the last years, this gene has been the focus of several studies linking it to carcinogenesis. For example, Hasegawa and collaborators have found that KCTD12 expression is associated with diagnosis and prognosis of gastrointestinal stromal tumors (GISTs). ${ }^{27}$ The possible mechanism by which the KCTD12 protein may exert an oncogenic push is facilitating the entrance of the cell in the $M$ phase and therefore promoting cell proliferation through the dephosphorylation of CyclinDependent Kinase 1 (CDK1, OMIM no. 116940). ${ }^{28}$ CDK1 is the cataIytic subunit of a protein kinase complex essential for $\mathrm{G} 2 / \mathrm{M}$ transition the aberrant expression of which is associated with PDAC. ${ }^{29}$

Considering the lack of bioinformatic data a mechanistic link between the SNP function and the gene expression is difficult to establish, a possible explanation for the association of 13q22.3 rs2328991 is that it could be associated with yet an unknown polymorphism possibly with a lower minor allele frequency (MAF) that could be the real culprit of the association. Fine mapping approaches have indeed successfully been used to identify rare variants close to GWAS findings. ${ }^{30}$

The increasing evidence of the involvement of pleiotropic regions in cancer etiology and the vicinity of a gene involved in cell cycle regulation make this finding potentially interesting.
For the three SNPs reported by Chen et al for which we had data we did not observe any statistically significant association. Possible reasons for these differences include the specific selection of candidate regions, the different analytical design of the studies.

An obvious strength of this report is its large sample size because with 3206 EOPC cases this is by far the largest study on biological determinants of this disease. A potential limitation of the study is the lack of data on epidemiologic risk factors since it is not possible to download covariate data from dbGaP. Considering that smoking behavior and family history of pancreatic cancer are strong risk factors for EOPC ${ }^{17}$ this could have led us to miss some associations or to not estimate correctly the associations we found. It is possible that the lack of adjustment for smoking and family history may cover the discovery of other potential SNPs. In addition, in the manuscript by Raimondi and colleagues the authors did not find markedly different percentage of familial cases in EOPC and VEOPC compared to NEOPC cases and a comparable effect of smoking in younger vs older cases. ${ }^{17}$ Therefore, it is unlikely that the patients in our study are enriched by familial cases or by heavy smoking.

Our result suggests the possible involvement of 13q22.3_rs2328991 in EOPC development in the largest study performed so far. However, it was not possible to find a mechanistic link between the variant and the function. These results need to be validated in larger datasets.

\section{ACKNOWLEDGEMENTS}

This work was partially supported by: intramural funds of DKFZFondazione Tizzi (www.fondazionetizzi.it) and by Fondazione Arpa (www.fondazionearpa.it) Daniele Campa. Péter Hegyi was supported by funding from the Hungarian Academy of Sciences (LP2014-10/2014) and by the Economic Development and Innovation Operative Programme Grant (GINOP 2.3.February 15, 2016-00048) of the National Research Development and Innovation Office. This work was also partially supported by the Ministry of Health of the Czech Republic grant no. NV19-03-00097 to Beatrice MohelnikovaDuchonova and Ministry of Health of the Czech Republic DRO (FNOI, 00098892) to Beatrice Mohelnikova-Duchonova. Charles University project ("Center of clinical and experimental liver surgery"no. UNCE/ MED/006 to P.S.), the Ministry of Health of the Czech Republic, project no. NV19-03-00097 to Viktor Hlavac and Martin Oliverius. The study was partially supported by Italian Ministry of Health grants (RC1803GA32) to the Division of Gastroenterology, Fondazione "Casa Sollievo della Sofferenza" IRCCS Hospital, San Giovanni Rotondo (FG), Italy and by the " $5 \times 1000$ " voluntary contribution. The ESTHER study was supported by a grant from the BadenWürttemberg state Ministry of Science, Research and Arts. Gabriele Capurso received a grant from Associazione Italiana per la Ricerca sul Cancro (AIRC) IG 17177.

\section{CONFLICT OF INTEREST}

Authors declare no conflict of interests. 


\section{DATA ACCESSIBILITY}

The PanScan genotyping data are available from the database of Genotypes and Phenotypes (dbGaP, study accession number phs000206.v5.p3). The PANDoRA primary data for this work will be made available to researchers who submit a reasonable request to the corresponding author, conditional to approval by the PANDoRA Steering Committee and Ethics Commission of the Medical Faculty of the University of Heidelberg. Data will be stripped from all information allowing identification of study participants.

\section{ETHICS APPROVAL}

Written informed consent was obtained from each participant. The PANDoRA study protocol was approved by the Ethics Commission of the Medical Faculty of the University of Heidelberg.

\section{ORCID}

Daniele Campa (D) https://orcid.org/0000-0003-3220-9944

Manuel Gentiluomo (D) https://orcid.org/0000-0002-0366-9653

Pavel Soucek (D) https://orcid.org/0000-0002-4294-6799

Francesca Tavano (D) https://orcid.org/0000-0002-8831-7349

Ben Schöttker (D) https://orcid.org/0000-0002-1217-4521

Maria Gazouli (D) https://orcid.org/0000-0002-3295-6811

Rudolf Kaaks (D) https://orcid.org/0000-0003-3751-3929

Federico Canzian (iD) https://orcid.org/0000-0002-4261-4583

\section{REFERENCES}

1. Bray F, Ferlay J, Soerjomataram I, Siegel RL, Torre LA, Jemal A. Global cancer statistics 2018: GLOBOCAN estimates of incidence and mortality worldwide for 36 cancers in 185 countries. CA Cancer J Clin. 2018;68:394-424.

2. Rahib L, Smith BD, Aizenberg R, Rosenzweig AB, Fleshman JM, Matrisian LM. Projecting cancer incidence and deaths to 2030: the unexpected burden of thyroid, liver, and pancreas cancers in the United States. Cancer Res. 2014;74:2913-2921.

3. Maisonneuve P, Lowenfels AB. Risk factors for pancreatic cancer: a summary review of meta-analytical studies. Int J Epidemiol. 2015;44:186-198.

4. Amundadottir L, Kraft P, Stolzenberg-Solomon RZ, et al. Genomewide association study identifies variants in the ABO locus associated with susceptibility to pancreatic cancer. Nat Genet. 2009;41:986-990.

5. Low $\mathrm{S}-\mathrm{K}$, Kuchiba A, Zembutsu H, et al. Genome-wide association study of pancreatic cancer in Japanese population. PLoS One. 2010;5:e11824.

6. Klein AP, Wolpin BM, Risch HA, et al. Genome-wide meta-analysis identifies five new susceptibility loci for pancreatic cancer. Nat Commun. 2018;9:556.

7. Campa D, Matarazzi M, Greenhalf W, et al. Genetic determinants of telomere length and risk of pancreatic cancer: a PANDoRA study. Int J Cancer. 2019;144:1275-1283.

8. Petersen GM, Amundadottir L, Fuchs CS, et al. A genome-wide association study identifies pancreatic cancer susceptibility loci on chromosomes 13q22.1, 1q32.1 and 5p15.33. Nat Genet. 2010;42:224-228.

9. Wu C, Miao X, Huang L, et al. Genome-wide association study identifies five loci associated with susceptibility to pancreatic cancer in Chinese populations. Nat Genet. 2011;44:62-66.

10. Wolpin BM, Rizzato C, Kraft P, et al. Genome-wide association study identifies multiple susceptibility loci for pancreatic cancer. Nat Genet. 2014;46:994-1000.

11. Campa D, Rizzato C, Stolzenberg-Solomon R, et al. TERT gene harbors multiple variants associated with pancreatic cancer susceptibility. Int J Cancer. 2015;137:2175-2183.
12. Childs EJ, Mocci E, Campa D, et al. Common variation at $2 \mathrm{p} 13.3$, $3 q 29,7 p 13$ and 17q25.1 associated with susceptibility to pancreatic cancer. Nat Genet. 2015;47:911-916.

13. Amundadottir LT. Pancreatic cancer genetics. Int J Biol Sci. 2016;12: 314-325.

14. Campa D, Capurso G, Pastore $M$, et al. Common germline variants within the CDKN2A/2B region affect risk of pancreatic neuroendocrine tumors. Sci Rep. 2016;6:39565.

15. Zhang $M$, Wang Z, Obazee $O$, et al. Three new pancreatic cancer susceptibility signals identified on chromosomes 1q32.1, 5p15.33 and 8q24.21. Oncotarget. 2016;7:66328-66343.

16. Piciucchi M, Capurso G, Valente R, et al. Early onset pancreatic cancer: risk factors, presentation and outcome. Pancreatology. 2015;15:151-155.

17. Raimondi S, Maisonneuve P, Lohr J-M, Lowenfels AB. Early onset pancreatic cancer: evidence of a major role for smoking and genetic factors. Cancer Epidemiol Biomarkers Prev. 2007;16:1894-1897.

18. McWilliams RR, Maisonneuve P, Bamlet WR, et al. Risk factors for early-onset and very-early-onset pancreatic adenocarcinoma: a pancreatic cancer case-control consortium (PanC4) analysis. Pancreas. 2016;45:311-316.

19. Ntala C, Debernardi S, Feakins RM, Crnogorac-Jurcevic T. Demographic, clinical, and pathological features of early onset pancreatic cancer patients. BMC Gastroenterol. 2018;18:139.

20. Ben-Aharon I, Elkabets M, Pelossof R, et al. Genomic landscape of pancreatic adenocarcinoma in younger versus older patients: does age matter? Clin Cancer Res. 2019;25:2185-2193.

21. Chen J, Wu X, Huang Y, et al. Identification of genetic variants predictive of early onset pancreatic cancer through a population science analysis of functional genomic datasets. Oncotarget. 2016;7:56480-56490.

22. Campa D, Rizzato C, Capurso G, et al. Genetic susceptibility to pancreatic cancer and its functional characterisation: the PANcreatic disease ReseArch (PANDoRA) consortium. Dig Liver Dis. 2013;45:95-99.

23. Boyle AP, Hong EL, Hariharan M, et al. Annotation of functional variation in personal genomes using RegulomeDB. Genome Res. 2012;22: 1790-1797.

24. Ward LD, Kellis M. HaploReg: a resource for exploring chromatin states, conservation, and regulatory motif alterations within sets of genetically linked variants. Nucleic Acids Res. 2012;40:D930-D934.

25. GTEx Project. GTEx portal. GTEx Anal. Release V6p (dbGaP Access. phs000424.v6.p1). 2017.

26. Dayem Ullah AZ, Oscanoa J, Wang J, Nagano A, Lemoine NR, Chelala C. SNPnexus: assessing the functional relevance of genetic variation to facilitate the promise of precision medicine. Nucleic Acids Res. 2018;46:W109-W113.

27. Hasegawa $\mathrm{T}$, Asanuma $\mathrm{H}$, Ogino J, et al. Use of potassium channel tetramerization domain-containing 12 as a biomarker for diagnosis and prognosis of gastrointestinal stromal tumor. Hum Pathol. 2013;44:1271-1277.

28. Zhong Y, Yang J, Xu WW, et al. KCTD12 promotes tumorigenesis by facilitating CDC25B/CDK1/Aurora A-dependent G2/M transition. Oncogene. 2017;36:6177-6189.

29. Khalid M, Idichi T, Seki N, et al. Gene regulation by antitumor miR204-5p in pancreatic ductal adenocarcinoma: the clinical significance of direct RACGAP1 regulation. Cancers (Basel). 2019;11:327.

30. Kote-Jarai Z, Saunders EJ, Leongamornlert DA, et al. Fine-mapping identifies multiple prostate cancer risk loci at $5 \mathrm{p} 15$, one of which associates with TERT expression. Hum Mol Genet. 2013;22:2520-2528.

How to cite this article: Campa D, Gentiluomo M, Obazee O, et al. Genome-wide association study identifies an early onset pancreatic cancer risk locus. Int. J. Cancer. 2020;147:

2065-2074. https://doi.org/10.1002/ijc.33004 